\title{
Some properties of meromorphically multivalent functions
}

\author{
Yi-Hui Xu' ${ }^{1}$ Qing Yang ${ }^{2}$ and Jin-Lin Liu ${ }^{2 *}$
}

* Correspondence: jlliu@yzu.edu.cn ${ }^{2}$ Department of Mathematics, Yangzhou University, Yangzhou, 225002, PR China

Full list of author information is available at the end of the article

\section{Abstract}

By using the method of differential subordinations, we derive certain properties of meromorphically multivalent functions.

2010 Mathematics Subject Classification: 30C45; 30C55.

Keywords: analytic function, meromorphically multivalent function, subordination

\section{Introduction}

Let $\Sigma(p)$ denotes the class of meromorphically multivalent functions $f(z)$ of the form

$$
f(z)=z^{-p}+\sum_{k=1}^{\infty} a_{k-p} z^{k-p} \quad(p \in N=\{1,2,3, \ldots\}),
$$

which are analytic in the punctured unit disk

$$
U^{*}=\{z: z \in C \text { and } 0<|z|<1\}=U \backslash\{0\} .
$$

Let $f(z)$ and $g(z)$ be analytic in $U$. Then, we say that $f(z)$ is subordinate to $g(z)$ in $U$, written $f(z) \prec g(z)$, if there exists an analytic function $w(z)$ in $U$, such that $|w(z)| \leq|z|$ and $f(z)=g(w(z))(z \in U)$. If $g(z)$ is univalent in $U$, then the subordination $f(z) \prec g(z)$ is equivalent to $f(0)=g(0)$ and $f(U) \subset g(U)$.

Let $p(z)=1+p_{1} z+\ldots$ be analytic in $U$. Then for $-1 \leq B<A \leq 1$, it is clear that

$$
p(z) \prec \frac{1+A z}{1+B z} \quad(z \in U)
$$

if and only if

$$
\left|p(z)-\frac{1-A B}{1-B^{2}}\right|<\frac{A-B}{1-B^{2}} \quad(-1<B<A \leq 1 ; z \in U)
$$

and

$$
\operatorname{Rep}(z)>\frac{1-A}{2} \quad(B=-1 ; z \in U) .
$$

Recently, several authors (see, e.g., [1-7]) considered some interesting properties of meromorphically multivalent functions. In the present article, we aim at proving some subordination properties for the class $\Sigma(p)$.

To derive our results, we need the following lemmas. 
Lemma 1 (see [8]. Let $h(z)$ be analytic and starlike univalent in $U$ with $h(0)=0$. If $g$ $(z)$ is analytic in $U$ and $z g^{\prime}(z) \prec h(z)$, then

$$
g(z) \prec g(0)+\int_{0}^{z} \frac{h(t)}{t} d t .
$$

Lemma 2 (see [9]. Let $p(z)$ be analytic and nonconstant in $U$ with $p(0)=1$. If $0<\mid$ $z_{0} \mid<1$ and $\operatorname{Re} p\left(z_{0}\right)=\min _{|z| \leq\left|z_{0}\right|} \operatorname{Re} p(z)$, then

$$
z_{0} p^{\prime}\left(z_{0}\right) \leq-\frac{\left|1-p\left(z_{0}\right)\right|^{2}}{2\left(1-\operatorname{Re} p\left(z_{0}\right)\right)}
$$

\section{Main results}

Our first result is contained in the following.

Theorem 1. Let $\alpha \in\left(0, \frac{1}{2}\right]$ and $\beta \in(0,1)$. If $f(z) \in \Sigma(p)$ satisfies $f(z) \neq 0\left(z \in U^{*}\right)$ and

$$
\left|\frac{z^{-p}}{f(z)}\left(\frac{z f^{\prime}(z)}{f(z)}+p\right)\right|<\delta \quad(z \in U),
$$

where $\delta$ is the minimum positive root of the equation

$$
\alpha \sin \left(\frac{\pi \beta}{2}\right) x^{2}-x+(1-\alpha) \sin \left(\frac{\pi \beta}{2}\right)=0,
$$

then

$$
\left|\arg \left(\frac{f(z)}{z^{-p}}-\alpha\right)\right|<\frac{\pi}{2} \beta \quad(z \in U) .
$$

The bound $\beta$ is the best possible for each $\alpha \in\left(0, \frac{1}{2}\right]$.

Proof. Let

$$
g(x)=\alpha \sin \left(\frac{\pi \beta}{2}\right) x^{2}-x+(1-\alpha) \sin \left(\frac{\pi \beta}{2}\right) .
$$

We can see that the Equation (2.2) has two positive roots. Since $g(0)>0$ and $g(1)<$ 0 , we have

$$
0<\frac{\alpha}{1-\alpha} \delta \leq \delta<1
$$

Put

$$
\frac{f(z)}{z^{-p}}=\alpha+(1-\alpha) p(z)
$$

Then from the assumption of the theorem, we see that $p(z)$ is analytic in $U$ with $p(0)$ $=1$ and $\alpha+(1-\alpha) p(z) \neq 0$ for all $z \in U$. Taking the logarithmic differentiations in both sides of (2.6), we get

$$
\frac{z f^{\prime}(z)}{f(z)}+p=\frac{(1-\alpha) z p^{\prime}(z)}{\alpha+(1-\alpha) p(z)}
$$


and

$$
\frac{z^{-p}}{f(z)}\left(\frac{z f^{\prime}(z)}{f(z)}+p\right)=\frac{(1-\alpha) z p^{\prime}(z)}{(\alpha+(1-\alpha) p(z))^{2}}
$$

for all $z \in U$. Thus the inequality (2.1) is equivalent to

$$
\frac{(1-\alpha) z p^{\prime}(z)}{(\alpha+(1-\alpha) p(z))^{2}} \prec \delta z .
$$

By using Lemma 1, (2.9) leads to

$$
\int_{0}^{z} \frac{(1-\alpha) p^{\prime}(t)}{(\alpha+(1-\alpha) p(t))^{2}} d t \prec \delta z
$$

or to

$$
1-\frac{1}{\alpha+(1-a) p(z)} \prec \delta z .
$$

In view of (2.5), (2.10) can be written as

$$
p(z) \prec \frac{1+\frac{\alpha}{1-\alpha} \delta z}{1-\delta z} .
$$

Now by taking $A=\frac{\alpha}{1-\alpha} \delta$ and $B=-\delta$ in (1.2) and (1.3), we have

$$
\begin{aligned}
\left|\arg \left(\frac{f(z)}{z^{-p}}-\alpha\right)\right| & =|\arg p(z)| \\
& <\arcsin \left(\frac{\delta}{1-\alpha+\alpha \delta^{2}}\right) \\
& =\frac{\pi}{2} \beta
\end{aligned}
$$

for all $z \in U$ because of $g(\delta)=0$. This proves (2.3).

Next, we consider the function $f(z)$ defined by

$$
f(z)=\frac{z^{-p}}{1-\delta_{z}}\left(z \in U^{*}\right) .
$$

It is easy to see that

$$
\left|\frac{z^{-p}}{f(z)}\left(\frac{z f^{\prime}(z)}{f(z)}+p\right)\right|=|\delta z|<\delta \quad(z \in U) .
$$

Since

$$
\frac{f(z)}{z^{-p}}-\alpha=(1-\alpha) \frac{1+\frac{\alpha}{1-\alpha} \delta z}{1-\delta z},
$$

it follows from (1.3) that

$$
\sup _{z \in U}\left|\arg \left(\frac{f(z)}{z^{-p}}-\alpha\right)\right|=\arcsin \left(\frac{\delta}{1-\alpha+\alpha \delta^{2}}\right)=\frac{\pi}{2} \beta .
$$

Hence, we conclude that the bound $\beta$ is the best possible for each $\alpha \in\left(0, \frac{1}{2}\right]$.

Next, we derive the following. 
Theorem 2. If $f(z) \in \Sigma(p)$ satisfies $f(z) \neq 0\left(z \in U^{*}\right)$ and

$$
\operatorname{Re}\left\{\frac{z^{-p}}{f(z)}\left(\frac{z f^{\prime}(z)}{f(z)}+p\right)\right\}<\gamma \quad(z \in U),
$$

where

$$
0<\gamma<\frac{1}{2 \log 2}
$$

then

$$
\operatorname{Re} \frac{z^{-p}}{f(z)}>1-2 \gamma \log 2 \quad(z \in U) .
$$

The bound in (2.14) is sharp.

Proof. Let

$$
p(z)=\frac{f(z)}{z^{-p}} .
$$

Then $p(z)$ is analytic in $U$ with $p(0)=1$ and $p(z) \neq 0$ for $z \in U$. In view of (2.15) and (2.12), we have

$$
1-\frac{z p^{\prime}(z)}{\gamma p^{2}(z)} \prec \frac{1+z}{1-z},
$$

i.e.,

$$
z\left(\frac{1}{p(z)}\right)^{\prime} \prec \frac{2 \gamma z}{1-z} .
$$

Now by using Lemma 1, we obtain

$$
\frac{1}{p(z)} \prec 1-2 \gamma \log (1-z) \text {. }
$$

Since the function $1-2 \gamma \log (1-z)$ is convex univalent in $U$ and

$$
\operatorname{Re}(1-2 \gamma \log (1-z))>1-2 \gamma \log 2 \quad(z \in U),
$$

from (2.16), we get the inequality (2.14).

To show that the bound in (2.14) cannot be increased, we consider

$$
f(z)=\frac{z^{-p}}{1-2 \gamma \log (1-z)} \quad\left(z \in U^{*}\right) .
$$

It is easy to verify that the function $f(z)$ satisfies the inequality (2.12). On the other hand, we have

$$
\operatorname{Re} \frac{z^{-p}}{f(z)} \rightarrow 1-2 \gamma \log 2
$$

as $z \rightarrow-1$. Now the proof of the theorem is complete.

Finally, we discuss the following theorem. 
Theorem 3. Let $f(z) \in \Sigma(p)$ with $f(z) \neq 0\left(z \in U^{*}\right)$. If

$$
\left|\operatorname{Im}\left\{\frac{z f^{\prime}(z)}{f(z)}\left(\frac{f(z)}{z^{-p}}-\lambda\right)\right\}\right|<\sqrt{\lambda(\lambda+2 p)} \quad(z \in U)
$$

for some $\lambda(\lambda>0)$, then

$$
\operatorname{Re} \frac{f(z)}{z^{-p}}>0 \quad(z \in U)
$$

Proof. Let us define the analytic function $p(z)$ in $U$ by

$$
\frac{f(z)}{z^{-p}}=p(z)
$$

Then $p(0)=1, p(z) \neq 0(z \in U)$ and

$$
\frac{z f^{\prime}(z)}{f(z)}\left(\frac{f(z)}{z^{-p}}-\lambda\right)=(p(z)-\lambda)\left(\frac{z p^{\prime}(z)}{p(z)}-p\right) \quad(z \in U) .
$$

Suppose that there exists a point $z_{0}\left(0<\left|z_{0}\right|<1\right)$ such that

$$
\operatorname{Re} p(z)>0\left(|z|<\left|z_{0}\right|\right) \text { and } p\left(z_{0}\right)=i \beta \text {, }
$$

where $\beta$ is real and $\beta \neq 0$. Then, applying Lemma 2 , we get

$$
z_{0} p^{\prime}\left(z_{0}\right) \leq-\frac{1+\beta^{2}}{2}
$$

Thus it follows from (2.19), (2.20), and (2.21) that

$$
I_{0}=\operatorname{Im}\left\{\frac{z_{0} f^{\prime}\left(z_{0}\right)}{f\left(z_{0}\right)}\left(\frac{f\left(z_{0}\right)}{z_{0}^{-p}}-\lambda\right)\right\}=-p \beta+\frac{\lambda}{\beta} z_{0} p^{\prime}\left(z_{0}\right) .
$$

In view of $\lambda>0$, from (2.21) and (2.22) we obtain

$$
I_{0} \geq-\frac{\lambda+(\lambda+2 p) \beta^{2}}{2 \beta} \geq \sqrt{\lambda(\lambda+2 p)} \quad(\beta<0)
$$

and

$$
I_{0} \leq-\frac{\lambda+(\lambda+2 p) \beta^{2}}{2 \beta} \leq-\sqrt{\lambda(\lambda+2 p)}(\beta>0) .
$$

But both (2.23) and (2.24) contradict the assumption (2.17). Therefore, we have Rep $(z)>0$ for all $z \in U$. This shows that (2.18) holds true.

\section{Author details}

${ }^{1}$ Department of Mathematics, Suqian College, Suqian 223800, PR China ${ }^{2}$ Department of Mathematics, Yangzhou University, Yangzhou, 225002, PR China

\section{Authors' contributions}

All authors read and approved the final manuscript.

\section{Competing interests}

The authors declare that they have no competing interests.

Received: 18 October 2011 Accepted: 16 April 2012 Published: 16 April 2012 


\section{References}

1. Ali, RM, Ravichandran, V, Seenivasagan, N: Subordination and superordination of the Liu-Srivastava linear operator on meromorphically functions. Bull Malays Math Sci Soc. 31, 193-207 (2008)

2. Aouf, MK: Certain subclasses of meromprphically multivalent functions associated with generalized hypergeometric function. Comput Math Appl. 55, 494-509 (2008)

3. Cho, NE, Kwon, OS, Srivastava, HM: A class of integral operators preserving subordination and superordination for meromorphic functions. Appl Math Comput. 193, 463-474 (2007)

4. Liu, J-L, Srivastava, HM: A linear operator and associated families of meromorphically multivalent functions J. Math Anal Appl. 259, 566-581 (2001)

5. Liu, J-L, Srivastava, HM: Classes of meromorphically multivalent functions associated with the generalized hypergeometric function. Math Comput Model. 39, 21-34 (2004)

6. Wang, Z-G, Jiang, Y-P, Srivastava, HM: Some subclasses of meromorphically multivalent functions associated with the generalized hypergeometric function Comput. Math Appl. 57, 571-586 (2009)

7. Wang, Z-G, Sun, Y, Zhang, Z-H: Certain classes of meromorphically multivalent functions. Comput Math Appl. $\mathbf{5 8}$ 1408-1417 (2009)

8. Suffridge, TJ: Some remarks on convex maps of the unit disk. Duke Math J. 37, 775-777 (1970)

9. Miller, SS, Mocanu, PT: Second order differential inequalities in the complex plane. J Math Anal Appl. 65, 289-305 (1978)

doi:10.1186/1029-242X-2012-86

Cite this article as: Xu et al: Some properties of meromorphically multivalent functions. Journal of Inequalities and Applications 2012 2012:86.

\section{Submit your manuscript to a SpringerOpen ${ }^{\odot}$} journal and benefit from:

- Convenient online submission

- Rigorous peer review

- Immediate publication on acceptance

- Open access: articles freely available online

- High visibility within the field

- Retaining the copyright to your article

Submit your next manuscript at $\boldsymbol{\wedge}$ springeropen.com 